\title{
Programa de autocuidado para el mejoramiento de la calidad de vida de atletas universitarios $^{1}$
}

\author{
Erika Duarte Carranza ${ }^{2}$ \\ Giorgianella Anderson Nedrick ${ }^{3}$
}

Institución: Universidad de Costa Rica

\section{COMO CITAR}

Duarte-Carranza, E. y Anderson-Nedrick, G. (Octubre, 2013). Programa de autocuidado para el mejoramiento de la calidad de vida de atletas universitarios. Rev. Enfermería Actual de Costa Rica, 25, 1-13. Recuperado de:: <http://www.revenf.ucr.ac.cr/deporte.pdf> ISSN 1409-4568

\section{RESUMEN}

El objetivo de este artículo es presentar los resultados de la intervención de Enfermería con los atletas de la Universidad de Costa Rica, por medio de un programa para la promoción de su autocuidado. Se partió de una investigación descriptiva exploratoria para diagnosticar las necesidades de autocuidado de una población de 40 atletas de distintas disciplinas deportivas colectivas. Se diseñó, ejecutó y evaluó el programa educativo para conocer el alcance que tuvo en los participantes. Los deportistas participantes alcanzaron los objetivos educativos propuestos y se mostraron positivos respecto de las actividades y contenidos del programa. Se concluye que es necesario implementar este tipo de intervención con los atletas universitarios desde la enfermería profesión capaz de asumir esta labor. Además existe un interés por parte del grupo de deportistas de continuar con estas actividades y mejorar así su salud general y su rendimiento en el deporte a largo plazo.

Palabras clave: autocuidado, deporte, atletas, actividad-física

\footnotetext{
${ }^{1}$ Fecha de recepción: 10 de noviembre 2013

${ }^{2}$ Enfermera. Trabajo independiente. Correo electrónico:negraedc8@gmail.com

${ }^{3}$ Enfermera. Trabajo independiente. Correo electrónico: giorgianella.g@gmail.com
} 


\title{
Self-care program for the improvement of the quality of life of university athletes ${ }^{1}$
}

Institution: University of Costa Rica

Ericka Duarte Carranza ${ }^{2}$

Giorgianella Anderson Nedrick ${ }^{3}$

\section{CITED}

\begin{abstract}
The objective of this article is to present the results of the intervention of nursing among athletes from the University of Costa Rica, through a self-care promotion program. It starts with an exploratory descriptive research to make a diagnosis of their self-care needs, with a population of 40 athletes from different group sports disciplines. It educational program was designed; executed and evaluated to know the extent that took in the participants. The participating athletes managed to reach the educational objectives proposed, leaving very positive comments regarding the activities and content of the program. It concluded that it is necessary to perform this kind of intervention with the university athletes, and nursing is a profession that is able to undertake this work. In addition there is interest by the athletes to continue with these activities and thereby improve their general health and their sport performance on the long term.
\end{abstract}

Keywords: athletes, Nursing, physical-activity, self-care, sports.

\footnotetext{
${ }^{1}$ Date of receipt: Noviembre 10, 2012

${ }^{2}$ Nurse. Independent work. E-mail: dlopezb.79@gmail.com

${ }^{3}$ Nurse. Independent work. E- mail: giorgianella.g@gmail.com
}

Date of acceptance: August 27, 2013 


\section{INTRODUCCIÓN}

En la actualidad, cada vez más personas eligen practicar actividad física regular y convertir el deporte en parte esencial de su vida. La práctica deportiva se define como todo movimiento de tipo voluntario planificado y repetitivo que busca mejorar o mantener una buena condición física o psíquica.

A partir de observaciones y vivencias se ha identificado que existe la necesidad de intervenir en la salud integral de las personas que practican deporte de manera regular y de alto rendimiento a nivel competitivo, para mejorar su calidad de vida y su rendimiento deportivo. De igual forma, existe la tendencia a pensar que una persona que practica deporte de alto rendimiento es automáticamente saludable y no necesita cuidar otros aspectos de su vida, más allá de su rendimiento físico y la prevención de lesiones.

Respecto de lo anterior, Fallas (2008) menciona que “...el deporte en sí representa un riesgo de sufrir lesiones en alta competencia, ámbito en el que deja de ser una actividad lúdica para convertirse en un escenario que propicia la aparición de problemas.” (p.1), no solo físicos, sino también orgánicos y psicológicos. Tales carencias revelan la necesidad de brindar atención integral a las personas que practican deporte de manera regular y de alto rendimiento, de lo cual se colige la relación que existe entre el autocuidado y la práctica deportiva, así como las formas en las que la Enfermería puede intervenir para mejorar la calidad de vida del deportista.

La investigación de antecedentes acerca de la relación entre autocuidado y práctica deportiva produjo un encuentro con una reciente especialidad de enfermería, enfocada en la práctica física denominada Enfermería Deportiva y de la Actividad Física, rama que nació en España alrededor de los años 90 y fue reconocida como tal por la Organización Colegial de Enfermería en 1997, entidad que establece que,

el profesional experto en enfermería deportiva es el responsable de la atención integral en el campo de la salud deportiva, un área que implica aspectos físicos, psíquicos y sociales, antes, durante y después de la actividad deportiva de cualquier modalidad y nivel competitivo, que conducen al bienestar y a la ausencia de dolencias en quienes la practiquen (Organización Colegial de Enfermería Española, 1997,p.3).

La incursión de enfermería en la actividad deportiva parte de un enfoque multidisciplinario e incluye una visión integral de la persona deportista, considerando que los hábitos de autocuidado de las personas distan de quienes no practican deporte competitivo.

A partir de lo anterior se plantea como objetivo el desarrollo de un programa de enfermería para la promoción del autocuidado en los deportistas de la Asociación Deportiva Universitaria, durante el primer semestre del 2011, cuyo propósito es solventar las necesidades de autocuidado de esa población y demostrar que la enfermería es capaz de incursionar en el cuidado y la atención en materia deportiva; tal como menciona (García, 2009, p.9), 
el profesional de enfermería se postula como el mejor colocado para prestar toda la atención necesaria al competidor, por ende, si se le proporcionan los conocimientos específicos de enfermería deportiva y el material necesario, se convierte en el profesional más completo para cuidar al deportista.

Además, existen elementos relevantes en la práctica de la enfermería deportiva, los cuales se apegan y se relacionan de manera directa con el concepto de autocuidado, mediante la educación sanitaria que,

se basa en la higiene deportiva, en potenciar aquellos factores que contribuyen a mejorar la actividad física y abarca desde la nutrición, ducha, aseo, hábitos de sueño, calzado, superficie de juego, prevención de lesiones y la hidratación. (...) contiene charlas acerca de la prevención de lesiones, primeros auxilios, alimentación y nutrición, higiene corporal y factores que contribuyen a mejorar la actividad (Martin, 2004, párr.5).

Si vemos, en enfermería la literatura sobre autocuidado es muy extensa, puesto que hay información, tanto del concepto en sí, como de la teoría de autocuidado de Orem, de los requisitos de autocuidado y de su relevancia en enfermería y de su aplicación para promover la salud y prevenir la enfermedad; sin embargo, la poca información relacionada con el ámbito del deporte competitivo representa un obstáculo, dado que no existe una integración documentada de estos elementos; la literatura existente abarca cada requisito por aparte, mas no los considera dentro de la atención integral del deportista, en lugar de ser tomados de una forma integral, como sucede en el autocuidado.

En la Escuela de Enfermería de la Universidad de Costa Rica, ningún Trabajo Final de Graduación ha abordado la temática del deporte o a las personas deportistas como tales. En nuestro país, se carece de publicaciones hechas por los profesionales, falencia aún más evidente en el área de enfermería y el deporte, donde es casi nula; unido a lo anterior, los profesionales relacionados con el tema del deporte y la atención en salud no desarrollan literatura al respecto y la bibliografía extranjera es limitada y de difícil acceso. Aparte, las distintas profesiones relacionadas con el deporte no integran los conocimientos de la enfermería ni el concepto de autocuidado.

En cuanto al concepto de autocuidado, Orem (1983) lo define como una actividad aprendida por los individuos, orientada a un objetivo y una meta: el cuidado de la persona; es la contribución constante de un adulto a su propia vida, salud y bienestar continuo, por medio de la satisfacción de los requisitos de autocuidado, los cuales, en algunos casos, son comunes a todo ser humano y otros son específicos y corresponden a los estados de desarrollo y de salud de la persona; así, los requisitos universales y las maneras de satisfacerlos pueden ser modificados por la edad, sexo o estado de desarrollo y salud; en este caso serán modificados por la práctica de deporte al nivel competitivo. 
El objetivo que justifica el porqué de analizar y discutir este tema parte de los riesgos por no mantener un debido autocuidado, pese a la práctica de la actividad deportiva.

En las observaciones realizadas a los atletas universitarios, se conocen condiciones que dificultan las prácticas de un correcto autocuidado; por ejemplo, la falta de conocimiento en algunos temas de alimentación e hidratación, prevención de lesiones y temas de comunicación asertiva; también en lo referente a la dificultad con los horarios de entrenamiento y de clase o trabajo y la falta de un mayor apoyo y acompañamiento por parte de entrenadores y entrenadoras, y personal de la Asociación Deportiva Universitaria (ADU) para llevar a cabo su autocuidado.

La idea que se pretende plasmar con este proyecto es que urge educar a la población deportista de la ADU en general acerca de los cuidados para mantener una mejor calidad de vida, así como concebir a la persona de forma integral y propiciar el autocuidado en las nuevas generaciones del deporte universitario.

Este trabajo se realizó por medio del sistema sustentador educativo de Orem a través de una metodología "educando a" y "orientando a", dado que promueve la participación de la persona involucrada respecto de la toma de decisiones, el control de sus acciones de cuidado y su comportamiento y la adquisición de los conocimientos y habilidades necesarias para mejorar su calidad de vida; en este caso, como se trabaja con deportistas, se busca que mejoren su rendimiento deportivo, objetivo que se vincula con el autocuidado del deportista, sus necesidades y los requisitos que se deben promover.

Para el deportista de élite la relevancia de la nutrición e hidratación adecuada, el valor del descanso en la práctica física, la influencia del estrés en el rendimiento deportivo, el manejo de lesiones y la necesidad de prevenirlas es imprescindible, razón por la que urge brindarle una orientación especial en cuanto a aspectos específicos en su autocuidado, no solo en nutrición, sino en aspectos como los que menciona Tobón (2003):

Entre las prácticas para el autocuidado se encuentran alimentación adecuada a las necesidades, medidas higiénicas, manejo del estrés, habilidades para establecer relaciones sociales y resolver problemas interpersonales, ejercicio y actividad física requeridas, habilidad para controlar y reducir el consumo de medicamentos, seguimiento para prescripciones de salud, comportamientos seguros, recreación y manejo del tiempo libre, diálogo, adaptaciones favorables a los cambios en el contexto y prácticas de autocuidado en los procesos mórbidos (p.40).

Lo anterior considera que un correcto autocuidado es directamente proporcional al rendimiento deportivo adecuado; por tanto, “....es impensable que un deportista tenga un rendimiento deportivo bueno si no se encuentra en las mejores condiciones de salud física, para lo cual el autocuidado es indispensable como estrategia de prevención y potenciador de una adecuada forma física". (Nava, 2009, p.1). 
El objetivo planteado en este trabajo fue desarrollar un programa de enfermería para la promoción de autocuidado en los y las deportistas de la Asociación Deportiva Universitaria durante el año 2012.

\section{MATERIALES Y MÉTODOS}

Este trabajo corresponde a un estudio de tipo descriptivo-exploratorio en su primera fase, con el fin de conocer así las necesidades de la población de atletas y obtener una visión general de la situación.

El trabajo se llevó a cabo en las instalaciones deportivas de la Universidad de Costa Rica, ubicadas en Sabanilla de Montes de Oca, las cuales corresponden a una zona delimitada, que forma parte de la extensión de terreno de la universidad denominado Finca \#3, cuya superficie es de 23 hectáreas, es amplia e incluye naturaleza y espacios al aire libre.

La población participante fue los atletas de la Asociación Deportiva Universitaria (ADU). Se incluyó deportistas de disciplinas colectivas, estudiantes activos de la Universidad de Costa Rica, quienes además contaban con los siguientes requisitos: edad entre los 17 y 30 años cumplidos, hombres y mujeres que practicaran un deporte de representación competitivos, pertenecientes a alguna de las cinco disciplinas colectivas por deporte-sexo: baloncesto, balonmano, fútbol, fútbol sala, voleibol; dentro de los criterios para la exclusión de este estudio está la negativa de las personas deportistas a participar. El total de participantes en la parte diagnóstica fue de 40 personas.

En la primera fase diagnóstica, se elaboró una matriz con variables de las que se obtuvieron los instrumentos de recolección de datos: el cuestionario autoadministrado y la guía de observación no participante. A partir de los resultados obtenidos de esta fase, se pasó a la fase de diseño del programa educativo de promoción de autocuidado, dentro de un diseño instruccional en el que se incorporaron objetivos educativos, contenidos, actividades de aprendizaje y evaluativas, así como materiales didácticos, hora y tiempo de desarrollo de las actividades.

Una vez desarrollado el programa, se procedió a la fase de implementación, ejecutada durante las jornadas de entrenamientos de los distintos equipos, por medio de charlas participativas, en sesiones grupales. Se implementó por medio de cinco temas, durante cinco sesiones, impartidas a cinco equipos elegidos por conveniencia, a saber los equipos de baloncesto femenino y masculino, voleibol femenino y masculino y balonmano masculino, debido a que cuentan con una sede de entrenamiento fija con facilidades para impartir las charlas. Cada uno de los equipos recibió las cinco charlas para un total de 25.

En cada una de las sesiones participaron entre 10 y 15 personas por equipo para un total estimado de 50 y 75 personas a lo largo de toda la implementación del programa.

Luego, se evaluó el programa de enfermería, su impacto en las personas participantes y sus alcances. Se empleó un instrumento de preguntas con escala tipo Likert, con el propósito de conocer los alcances de los 


\section{Revista Electrónica Enfermería Actual en costa Rica}

contenidos del programa en la labor deportiva. Se aplicó al término de la capacitación para conocer las impresiones, los beneficios obtenidos, así como los comentarios de los participantes para considerarlas dentro de las conclusiones y recomendaciones finales.

Los datos se procesaron con el programa Microsoft Office Excel 2007® y se graficaron los datos más relevantes para facilitar la presentación. Luego se procedió a analizarlos para diseñar la capacitación y se agregaron los datos más relevantes obtenidos a partir de la observación no participante.

Los resultados de la implementación del programa se obtuvieron al final de cada una de las sesiones, a través de lluvia de ideas, preguntas y respuestas, y aclaración de dudas por parte de los participantes, para conocer el alcance en específico de la sesión y el cumplimiento de los objetivos educativos de cada encuentro.

\section{Consideraciones éticas}

Se solicitó mediante cartas a la ADU su aprobación para desarrollar el programa. Además, se aplicó el consentimiento informado, elaborado por las investigadoras (se explican los lineamientos generales de la participación en la práctica dirigida, según la normativa universitaria dada por la Vicerrectoría de Investigación), considerando los criterios de autonomía, beneficencia, no maleficencia, integridad y fidelidad.

\section{RESULTADOS}

Entre los datos generales se encontró que en la población encuestada prevalece la participación femenina (57,5\%), aun cuando son solo dos equipos femeninos (voleibol y baloncesto). La mayoría de las áreas o facultades de la Universidad son representadas por al menos un o una estudiante que practica deporte a nivel competitivo. De los atletas, 28 cursan el $3^{\text {er }}$ y $4^{\circ}$ año de carrera universitaria, mientras que el resto cursa el $1^{\circ}$ y $2^{\circ}$ año de carrera. Más de la mitad (67.5\%) han formado parte del equipo universitario por más de un año.

En cuanto al tiempo de estudio, la cantidad invertida es mucha, lo cual limita el tiempo para actividades propias del autocuidado, tales como ingerir una alimentación adecuada, disponer de tiempo libre y recreativo, así como de espacios para favorecer la salud mental.

La mayoría (un 37.5\%) acude cinco días por semana a estudiar a la universidad, seguido de un $35 \%$ que asiste cuatro días, datos a partir de los que se infiere que más del $70 \%$ de las personas deportistas encuestados matriculan bloque completo de cursos de sus respectivas carreras; luego, alrededor de un $57 \%$ de las personas participantes invierten más de 16 horas semanales en asistir a lecciones, seguido de un $12 \%$ que va de 12 a 16 horas.

Respecto de las prácticas deportivas, estas demandan una valiosa inversión de tiempo que, junto al tiempo de estudio, significa una demanda de autocuidado aumentada, máxime que un $65 \%$ de los participantes invierten entre 3 y 6 horas en el desarrollo de la práctica del deporte, mientras que un 37.5\% dedican entre 1 y 3 horas de 
actividad física extra por semana, principalmente con sesiones de pesas, acondicionamiento físico y baile, seguido de un $27.5 \%$ que dice realizar entre 3 y 6 horas y un $25 \%$ que lo hace de 6 a 9 horas. Un $35 \%$ de la población, además de ser estudiantes y atletas, también trabaja.

En relación con los conocimientos de autocuidado, la mayor parte de la población encuestada muestra conocimiento del tema, así como de sus implicaciones y aspectos generales. Los participantes coinciden en que un correcto autocuidado es directamente proporcional para su salud general y su rendimiento deportivo; no obstante, al actuar, se evidencian déficits.

En cuanto a los hábitos de autocuidado en alimentación se evidenció que un $84 \%$ tiene hábitos alimenticios regulares o buenos, un 32\% realiza cinco o más tiempos de comida al día. En días de competencias, un $45 \%$ mantiene los mismos hábitos alimenticios, aunque un 17\% ingiere una mala o muy mala alimentación.

En lo referente a la hidratación, es evidente que un $40 \%$ bebe menos de los requerimientos diarios de agua, además de que los tiempos de hidratación durante los entrenamientos son deficientes. Luego, un $67 \%$ de participantes no realiza una rehidratación adecuada posterior al ejercicio; por consiguiente, una gran mayoría se encuentran en un déficit de su autocuidado en la ingesta de agua. En cuanto a la ingesta de bebidas alcohólicas, un $70 \%$ bebe en ocasiones, un $25 \%$ nunca ha bebido y un $5 \%$ bebe con frecuencia.

Un porcentaje alto de personas ( $95 \%$ de la población total encuestada) cuenta con un factor positivo, no fuman, mientras que un 5\% sí lo hace. Lo anterior es destacable, dado que es un factor protector para el requisito universal de autocuidado para la ingesta de aire.

En relación con el sueño y descanso, los participantes afirman que les queda poco tiempo para descanso; es decir, presentan un déficit en ese rubro. Un $60 \%$ refiere dormir un promedio de 6 a 8 horas con una calidad del sueño buena y muy buena, lo cual demuestra que presentan un patrón de sueño adecuado.

En cuanto a las relaciones interpersonales dentro del equipo, un $83 \%$ las considera como buenas o muy buenas, mientras que un $87,5 \%$ refiere contar con el apoyo de sus compañeros de equipo.

En las situaciones generadoras de estrés, los participantes clasificaron que en primer lugar se encuentra el estudio, seguido del bajo rendimiento deportivo (75\%), las lesiones sufridas, problemas familiares y, por último, los problemas sentimentales.

En el aspecto de prevención de lesiones deportivas, un 50\% ha sufrido alguna lesión deportiva en el último año. Entre los participantes las lesiones más frecuentes son fisuras en falanges, esguinces de tobillo, contracturas, esguinces de rodilla. En los atletas que han sufrido lesiones, el 25\% autoadministra su tratamiento y el $47.5 \%$ se automedica (consumir medicamentos para el dolor, antiinflamatorios, relajantes musculares, sin indicación médica) en caso de una lesión. De la población total, un $85 \%$ conoce cómo prevenir las lesiones deportivas, aunque hay evidencia de mal uso e incumplimiento de estas estrategias. 
Respecto de la implementación del programa, se llevó a cabo durante los meses de junio y julio del 2012 y fue impartido a los equipos de baloncesto femenino y masculino, voleibol femenino y masculino y balonmano masculino. Fue divido en cinco temas principales, durante cinco sesiones, a saber alimentación e hidratación, lesiones deportivas ( 2 sesiones), primeros auxilios en el deporte y salud mental en el deporte. En cada sesión se llevó a cabo con una metodología de charlas dinámicas, participativas, entre 20 y 25 minutos, previo a cada entrenamiento, para un total de 125 minutos.

En la evaluación final, tras implementar el programa, fue evidente que los participantes lograron un alto nivel de aprovechamiento, en el que obtuvieron nuevos conocimientos de mucho provecho y aplicables a su estilo de vida y salud general. Los participantes fueron muy colaboradores y se mostraron muy interesados en que este tipo de programas se implementen de manera periódica, lo cual permitió que se cumplieran los objetivos del programa; por tanto, se generó un mensaje de positivismo en los participantes.

\section{DISCUSIÓN}

Es posible ver que en relación con el tiempo de estudio en días por semana y horas semanales, la cantidad invertida para esta actividad es mucha, lo cual limita el tiempo que se invierte en actividades propias del autocuidado.

Conforme aumentan los años de carrera, aumenta el tiempo de práctica deportiva que, a su vez, incrementa las necesidades de autocuidado, dado que demanda más tiempo de actividad y se resta tiempo libre y tiempo para cumplir con las demandas de autocuidado.

En relación con la cantidad de tiempo invertido en prácticas deportivas, tanto en el equipo de representación como en actividades extra, es notable que los atletas dedican mucho tiempo a esos rubros, a los cuales se les debe agregar el tiempo de estudio; por tanto, se limitan las posibilidades de cumplir con un correcto autocuidado; es decir, hay una demanda de autocuidado aumentada que evidencia la necesidad de reforzar las habilidades para cumplir con un correcto autocuidado.

Existe un número notorio de atletas que además de ser estudiantes universitarios activos y deportistas de alto rendimiento, también trabajan, característica que limita el tiempo del autocuidado y aumenta el riesgo para adquirir un déficit en alguno de sus requisitos de autocuidado. En este contexto, la enfermería debe identificar las situaciones de riesgo, para corregir lo necesario en el tiempo oportuno y reforzar las áreas que así lo requieran, mediante programas educativos y de guía enfocados en el autocuidado.

Existe un conocimiento acerca de los elementos de autocuidado que deben practicar los deportistas, los cuales difieren de las personas que no son atletas; por ejemplo, como menciona González-Gross et al. (2001), la práctica regular de ejercicio físico a una intensidad media-alta, como la ejecutan los deportistas profesionales o de alto rendimiento, conduce a una serie de cambios metabólicos y fisiológicos que marcan las diferencias 
nutricionales respecto de las personas sedentarias o que practican deporte con una intensidad menor, razón por la que la enfermería desempeña un papel relevante al incorporar ajustes en las necesidades de los atletas con el fin de brindar acciones integrales según la población.

En la alimentación, debe prestarse mucha atención a los hábitos alimenticios que tengan para que puedan suplir sus requerimientos nutricionales, un requisito de autocuidado que es de los más influyentes en el rendimiento deportivo, el cual "...depende en gran medida de la dieta que se consume antes, durante y después del entrenamiento y competencia" (Halcones, s.a., p. 1). Dado lo anterior, un déficit en este requisito de autocuidado puede entorpecer la preparación para los entrenamientos deportivos, así como en los días de competencia, dado que no importa cuánto se prepare el deportista a nivel técnico: una mala alimentación imposibilita un adecuado rendimiento deportivo.

En cuanto a la hidratación, el agua constituye una prioridad durante el ejercicio y en las actividades regulares; por ende, el deportista no debe olvidar el agua dentro de sus hábitos de autocuidado. Al respecto, es necesario reforzar la relevancia de un correcto autocuidado en la ingesta de líquidos para que el balance hídrico del individuo no se vea afectado y, a la larga, tampoco su rendimiento deportivo ni su salud en general.

Un gran porcentaje de atletas consumen alcohol de manera ocasional, una de las drogas lícitas más usadas por los deportistas por razones sociales, como menciona Umaña (2009), aunque su consumo antes o después del entrenamiento o la competencia, no mejora el rendimiento, más bien puede perjudicarlo. El rendimiento psicomotor y el juicio son los más afectados, puesto que el alcohol puede deshidratar, retrasar los reflejos, bajar el azúcar en la sangre y disminuir el rendimiento; además, afecta el proceso de recuperación del cuerpo posterior a la práctica deportiva, lo cual pone un estrés extra al organismo que perjudica su capacidad de responder y de recuperarse para la siguiente práctica física.

La mayoría de sustancias estimulantes que ingieren las personas deportistas universitarias se relacionan con bebidas energéticas, suplementos de cafeína, y sustancias para mantenerse despiertos, lo cual se liga con los hábitos de estudio de cada atleta, práctica muy extendida entre un pequeña cantidad de estudiantes universitarios en general, mas no es recomendable, máxime que, como agregan Araya y Campos (2008), no genera beneficio alguno al rendimiento deportivo y, por lo general, este tipo de bebidas producen problemas gastrointestinales si son consumidas previo a la actividad física. No obstante,

muchos atletas utilizan las bebidas energéticas por su efecto estimulante para mejorar la concentración, la atención y el tiempo de reacción. Los datos para respaldar este efecto son limitados pero brindan cierta evidencia para defender muchas de estas afirmaciones empíricas de los atletas ( 
A pesar de la insistencia en su uso, su ingesta de ninguna manera sustituye un adecuado autocuidado en cuanto al sueño y descanso, como estrategia para mejorar la atención y concentración.

Por otro lado, el debe prestar especial interés al poder reparador que ofrece un adecuado patrón de sueño y descanso, puesto que "se ha remarcado desde un punto de vista absolutamente aplicado y práctico, la relevancia del sueño (como una actividad potencialmente regeneradora mental y física) en distintas actividades físicas y deportivas" (García- Aguado, Cuartero, Calabria, Jiménez, \& Pérez, 2003. p. 183). Un sueño reparador ayuda a que las facultades mentales sean más productivas y a que el cuerpo se autorrepare para ejecutar las actividades diarias; luego, en condiciones de entrenamiento y competición es elemental para ayudar al cuerpo a recuperarse tanto en lo físico como en lo mental; por ende, cumplir con patrones de sueño y descanso adecuado es muy relevante para los deportistas.

En el ámbito de las relaciones interpersonales, estas son indispensables en los equipos para que se logre el éxito, debido a que optimiza el rendimiento deportivo y la resolución de problemas. Si existe una buena salud mental e implementación de una comunicación asertiva, las personas podrán adquirir un nivel de bienestar mental a la hora de ejecutar sus actividades diarias tanto dentro como fuera de su equipo deportivo. Un amplio porcentaje de deportistas considera las relaciones entre los equipos de representación universitaria como buena; no obstante, entre los equipos debe existir una relación socioafectiva óptima, debido a que forman parte de la misma casa de estudios y comparten las mismas instalaciones de juego y práctica. Como menciona García (2003), el rendimiento deportivo de un equipo depende de manera directa de la «cohesión» grupal, uno de los factores psicosociales más relevantes para un rendimiento deportivo, además, las relaciones interpersonales positivas afectan la salud mental de todo ser humano.

En el ámbito de las situaciones generadoras de estrés se debe considerar que para ser parte de los equipos de representación universitaria, primero, hay que cumplir con varios requisitos entre los que se menciona ser estudiantes activos de la universidad con una carga académica mínima de créditos por semestre, buen rendimiento académico y deportivo, inversión de horas en práctica deportiva semanal, condiciones que generan estrés en los atletas. Respecto del estrés y el rendimiento deportivo, Tallano (2008) define el primero como un desequilibrio entre la persona, el entorno y la demanda:

El sujeto en situación deportiva percibe una demanda; cuando la demanda supera la capacidad de dar respuesta se produce un incremento de la ansiedad, hay reacciones fisiológicas y psicológicas que influyen en el rendimiento (...) Hay situaciones que estresan al sujeto en situación deportiva, las cuales se relacionan con la vida personal, personalidad, competencia, entrenamiento. Otro factor fundamental que puede ser estresante es la comunicación con los distintos actores del deporte: dirigentes, prensa, árbitros, quienes se exponen a constantes situaciones de evaluación (Tallano, 2008, párr.2). 
De lo anterior se colige que el estrés es un determinante en la práctica deportiva que se traduce en un rendimiento ineficaz; por tanto, es imprescindible abordar desde los principios del autocuidado lo referente al manejo de situaciones de estrés y solución de conflictos por parte de enfermería, encargada de brindar las pautas principales para solucionar o minimizar los conflictos.

Luego, en lo que se refiere a la prevención de lesiones, se demuestra que un alto porcentaje de la población deportista conoce cómo cuidarse en caso de lesionarse; desde la enfermería se pretende que el conocimiento aprendido se lleve a la práctica diaria, de modo que las estrategias de prevención no sean relegadas a un segundo plano, máxime que “...las lesiones necesitan no solo un correcto diagnóstico, una atención y cuidados atentos y un tratamiento adecuado, sino también una prevención que genere el sentimiento de bienestar y una mejor calidad de vida derivados de la práctica deportiva" (Martín, 2004, p.55 ).

Al finalizar, el programa fue bien aprovechado por los atletas universitarios, quienes se mostraron interesados por extender su implementación y que se imparta de manera regular para que se abarquen más cantidad de temas y se profundice en estos; lo cual precisa de un lugar adecuado en el que capte por completo la atención de los atletas, de una manera más práctica y activa.

La interacción con los atletas propició el desarrollo de habilidades en el área de la Enfermería Deportiva, la cual despertó el interés de los participantes de obtener nuevos conocimientos que beneficien su salud y la práctica deportiva, situación que se repitió con los cuerpos técnicos (entrenadores y asistentes), quienes se mostraron muy preocupados e interesados en los contenidos y objetivos de este programa de enfermería.

También es evidente que en el ambiente deportivo, y más aún en uno universitario, es vital la intervención continua de un profesional capacitado en enfermería del deporte para que las personas reciban un abordaje integral continuo y no solo cuando sufren lesiones.

\section{CONCLUSIONES}

Los programas para el autocuidado favorecen la prevención de los déficits y refuerzan los hábitos positivos de autocuidado, brindando educación, orientación y acompañamiento, de manera que el abordaje interactivo y práctico, con temáticas variadas estimulen el proceso de aprendizaje y el aprovechamiento y que propicie las relaciones interpersonales, tanto entre los compañeros del equipo, como entre el equipo y su respectivo cuerpo técnico.

Desde la enfermería es posible contribuir en la orientación de dicho grupo acerca de las estrategias para lograr el autocuidado y optimizar sus hábitos saludables, mas si se considera la formación educativa que aborda Enfermería, la cual incluye un enfoque integral y holístico, con contenidos que abarcan muchas esferas de la vida del ser humano para que sea capaz de mantener un funcionamiento adecuado y que, de esta manera, pueda cumplir su fin último como deportista y alcance un rendimiento deportivo óptimo. 


\section{Revista Electrónica Enfermería Actual en costa Rica}

\section{REFERENCIAS}

Araya, G., Campos, C, (2008). Bebidas energéticas y rendimiento deportivo. Recuperado de: http://www.edufi.ucr.ac.cr/pdf/trans/Bebidas\%20energ\%E9ticas\%20y\%20rendimiento\%20f\%EDsico.pdf

Fallas, N. (2008). Factores Psicológicos Relacionados con la Incidencia y Recuperación de las lesiones Deportivas en Futbolistas. Tesis de Postgrado en Salud Integral y Movimiento Humano con mención en Rendimiento Deportivo para optar por el titulo de Magíster Scientiae: Universidad Nacional, Heredia, Costa Rica.

García, J. (2009). Atención de Enfermería en competiciones deportivas: el botiquín de campo. Revista ROL de enfermería, 31 (12), 8-12.

García, A., Vicens, P. (2003). La psicología del equipo deportivo. Cooperación y rendimiento. Revista de Psicología del Deporte. 6 (2), 79-89.

García, A., Aguado, F., Cuartero, J., Calabria, E., Jiménez, R., Pérez, P. (2003). Sueño, descanso y rendimiento en jóvenes deportistas de competición. Revista de Psicología del Deporte. 12 (2), 181-195.

González, M., Mesa, J., Ruiz, J., Castillo, M. (2001). La nutrición deportiva en la práctica deportiva: adapatación de la pirámide nutricional a las características de la dieta del deportista. Archivos Latinoamericanos de Nutrición, 321-331.

Halcones, A. (s.a.). Alimentación en el Deportista. Generalidades. Veracruz: Univ. Veracruzana.

Hoffman, J. (2010). Caffeine and Energy Drinks. Strength \& Conditioning Journal, 32(1), 15-20.

Martín, A. (2004). La enfermería del deporte en las estrategias preventivas de atención (aplicadas al fútbol). Recuperado de: www.canariasdeportiva.com/index.php?id=detalle.php\&mid=alimentacion.php\&a $=$ todo\&detalle $=6809$

Nava, M. (2009). Factores del Entrenamiento Deportivo. México D.F.: Recuperado de http://centroculturalneza.galeon.com/factdep.pdf.

Organización Colegial de Enfermería Española. (1997). Resolución $N^{\circ} 7 / 97$ : Aspectos del ejercicio profesiona, en el ámbito de la enfermería deportiva y de la actividad física. España: Consejo General.

Orem, D. (1983). Normas prácticas de Enfermería. Madrid: Pirámide Ediciones.

Tallano, L. (2008). Estrés y rendimiento deportivo. Federación Entrerriana de Natación. Recuperado de http://www.fen.org.ar/capacitacion/estres.htm

Tobón, O. (2003). El Autocuidado una habilidad para vivir. Hacia la Promoción de la Salud , 37-49. Recuperado de http://bases.bireme.br/cgibin/wxislind.exe/iah/online/?IsisScript=iah/iah.xis\&src=google\&base=LILACS\&lang=p\&nextActi on=lnk\&exprSearch=479433\&indexSearch=ID.

Umaña, M. (2009). Alcohol y rendimiento deportivo. NUTRIDEP. S.A. Recuperado de CR Deportes: http://www.crdeportes.com/wordpress/?p=192 